\title{
Training system of percutaneous endoscopic lumbar discectomy in China
}

\author{
De-Hong Yang \\ Department of Spinal Surgery, Nanfang Hospital, Southern Medical University, Guangzhou 510515, Guangdong, China.
}

Correspondence to: Dr. De-Hong Yang, Department of Spinal Surgery, Nanfang Hospital, Southern Medical University, Guangzhou 510515, Guangdong, China. E-mail: drmyang@yahoo.com

How to cite this article: Yang DH. Training system of percutaneous endoscopic lumbar discectomy in China. Mini-invasive Surg 2017;1:106-8.

Article history: Received: 18 Apr 2017 Accepted: 4 Jul 2017 Published: 30 Sep 2017

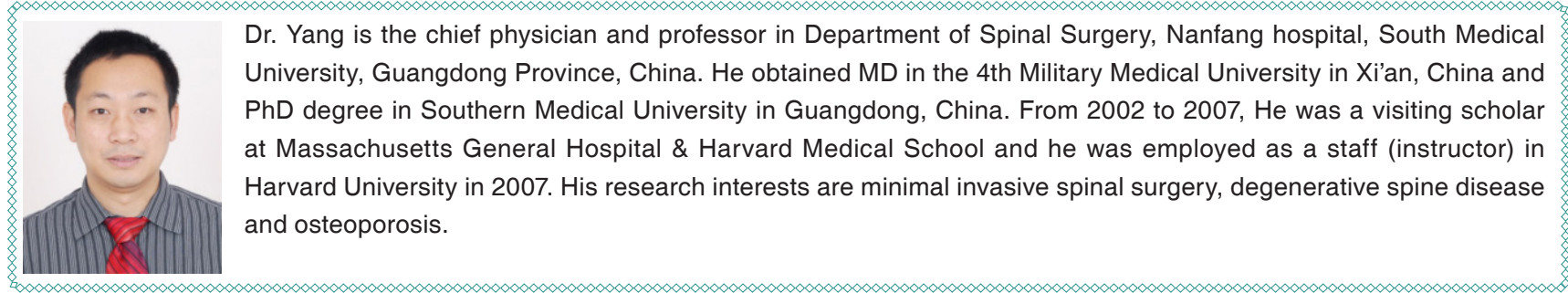

Percutaneous endoscopic lumbar discectomy (PELD) is by far the most minimally invasive technique for lumbar disc herniation. The small incision, short recovery time, relatively low cost and low surgical morbidity make this technique attractive for both doctors and patients. However, the technique is still unfamiliar to doctors, even those with many years of experience in spine surgery. In brief, the core of this technique is "placing the working cannula in right location and forceping out the herniated disc" based on our own experience. However, surgeons, especially beginners, sometimes get "lost" in their operations, which can result in complications such nerve damage, disc fragment left over, etc.

For many surgeons, the PELD technique is challenging to learn because the training process is different from open surgery. In the open operation, the close collaboration between responsible and assistant doctors gives the assistant a lot of opportunities to practice during the operation. PELD operation is an one person performance, the forcep holder deal with everything and assistant does not have any real practice. However, once the beginner stands by the patient and holds the instruments, he has to rely mostly on himself. Consequently, the initial learning process is a technical challenge that it is thought to be insurmountable for some surgeons. The slow learning curve, long hands-on training time, together with insufficient training source, discourages doctors to adopt PELD technique. Therefore, quite a few doctors chose open surgery rather than PELD to treat lumbar disc herniation, because they were more confident with their open surgery techniques instead of PELD. License (https://creativecommons.org/licenses/by/4.0/), which permits unrestricted use, distribution, and reproduction in any medium, as long as the original author is credited and the new creations are licensed under the identical terms.

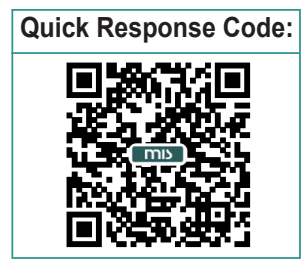


After two decades of developing the surgical approach and visible technique, Yeung ${ }^{[1]}$ introduced his "insideout" technique (YESS) with a rigid rod-lens, water flow-integrated, and multichannel spinal cannula that combined a camera, light and the working channel together. He introduced this technique into China in 1997. More doctors in China began to learn the YESS technique, and they began to practice this technique more routinely. They became mentors for other surgeons in China who wanted to learn the technique. In 1999, Hoogland et al. ${ }^{[2]}$ started a new technique of a lateral transforaminal approach to the spinal canal (THESSYS). The THESSYS technique was introduced to China in $2007^{[3]}$. After mastering the PELD technique, surgeons began to spread this technique, and a formal training system was soon developed.

Before the PELD training program, minimally invasive spinal surgery workshops involving MED technique appeared in China as early as 2004. In 2007, PELD technique was introduced in seminars. In 2010, PELD cadaver workshop advertisements appeared in meeting notices. Currently, more than 20 seminars were held in China annually. As other surgical technique trainings, the training of PELD for Chinese doctors was in two stages. The first consisted of training abroad in which pioneers were educated abroad and returned to their home country to start practice. The second was training at home in which overseas returnees introduced PELD technique and held workshops to spread the techniques. For a surgeon without a background in endoscopic surgery, seminar, cadaver workshop and hands on practice are the stepwise way to learn the technique. The important areas that help the surgeon to understand the PELD technique include a clear understanding of the anatomical structure and image pictures, accurate judgment of different tissue under endoscopy, and correct 3D positional imprints. Therefore, training and learning are heavily focused on these three aspects.

For a typical training class, teaching modules generally include an introduction of PELD technique, working cannula placement, endoscopic performance, and pitfalls that might be encountered. After lectures, cadaver workshop let each attendee practice the working cannula insertion and practice operations under endoscopy. An operation demonstration with instant explanation would come before or after workshop. Then, before practicing operations on patients, an intensive watching and hands-on operations needs to be finished. Many surgeons have expressed interest in attending a mid-career training program to focus on the PELD technique. They would have an opportunity to get hands-on operation training during that period. In addition, some physicians have expressed a desire to to invite experts to their local hospitals in order to have hands on demonstrations.

Chinese teachers have invented a couple of methods to boost the learning ${ }^{[4]}$. They guide the trainees to combine X-ray, magnetic resonance imaging and computed tomography images to $3 \mathrm{D}$ models to help the trainees build $3 \mathrm{D}$ images. The learning curve is 60 operations for the surgeon to be skilled to perform PELD ${ }^{[5]}$. In a transforaminal approach, the $L 4 / 5$ technique was easier to master than $L 5 / S 1^{[6]}$.

In order to standardize the application of minimal invasive spinal surgery in China, a Spinal Endoscopic Diagnosis and Treatment Management Standards (edition 2013) was established by National Health and Family Planning Commission in $2013^{[7]}$. It established detailed requirements for medical institutions, doctors and ancillary staff. It also set the standard for endoscopic diagnosis and treatment. Most importantly, the detailed requirements of medical education and training bases for spinal endoscopic activities were clearly defined. The education bases must be:

1. Class $3 A$ ranked hospitals;

2. No less than 10 years experience in spinal diseases treatment, with grade 4 (highest) surgical ability to perform spinal endoscopic activities; no less than 1,500 spinal cases were surgically treated in the past 3 years, and 50 cases of endoscopic operations annually;

3. No less than 2 doctors have grade 4 spinal endoscopic surgery ability, and at least 1 is a consultant doctor;

4. The institution fulfills the entire requirement including staffs, techniques, instruments and facilities;

5. The institution has held national spinal endoscopy meeting or finished national continuing medical education program.

After establishment, it served as a solid protocol, but updates are absolutely required, especially as more and more doctors obtained the ability to perform good PELD.

In China, physicians not working in spinal surgery units have performed the PELD technique in treating lumbar disc herniation. It is difficult to determine the training qualifications of the doctors who perform PELD in pain clinics. An Internet search of renowned physicians in the Pain department with their showed that some doctors switched from the Orthopedic Department and some had switched from the Anesthesia Department. In the beginning, they all received training from an orthopedist and now they collaborate with spine orthopedist to open training programs. The Association of Chinese Spine 
Pain and Minimal Invasive Technique was established in 2016 and provided a new communication platform for all kind of doctors who performed PELD technique.

Although progress has been achieved, short-term training programs were still not enough for attendees to start practicing PELD. Even after a couple of training classes, many doctors were hesitant to undertake their first operation. This reason can be summed up by the Chinese expression: "easy when watching and hard while doing". In the future, the training system should focus on "hands on" practice in the operating room.

The PELD technique has become more and more widely accepted by doctors and patients. New indications for the procedure include: spinal canal stenosis, infections, and tumors. In addition, new instruments have been developed. Systematic training or re-training of surgeons is necessary. First, the trainees need to be classified according their background and experience on endoscopic practice. For example, spinal surgeons are familiar with anatomical structure of lumbar spine and more skilled in handling possible pitfalls whereas anesthetists are familiar with punctures. Some doctors come to learn from how to perform the technique and others come to resolve problems in their practice. Accordingly, the teaching methods and courses need to fulfill the different expectations. Second, training system needs to be focused on both quantity and quality. Besides short-term training, trainees and trainers should also focus on long-term hands on clinical training. Third, training needs to be separated into basic and advanced stages. Two types of training facilities should be established. Certificated regional training center focused on short-term training including lecture, cadaver practice and operation observation. Certificated clinics focused on hands-on training. Fourth, the old protocol needs to be revised. Strict rules should be applied to standard certification system to guide the training facilities and to make sure only qualified doctors perform PELD.

\section{DECLARATIONS}

\section{Authors' contributions}

D.H. Yang contributed solely to the paper.

\section{Financial support and sponsorship}

None.

\section{Conflicts of interest}

There are no conflicts of interest.

\section{Patient consent}

Not applicable.

\section{Ethics approval}

Not applicable.

\section{REFERENCES}

1. Yeung AT. Minimally invasive disc surgery with the Yeung Endoscopic Spine System (YESS). Surg Technol Int 1999;8:267-77.

2. Hoogland T, Schubert M, Miklitz B, Ramirez A. Transforaminal posterolateral endoscopic discectomy with or without the combination of a low-dose chymopapain: a prospective randomized study in 280 consecutive cases. Spine (Phila Pa 1976) 2006;31:E890-7.

3. Zhang XF, Zhang L. The past, present and future of spine endoscopic technology in China. Chin J Pain Med 2015;21:81-5. (in Chinese)

4. Wang HW, Li CQ, Zhou Y. Application of imaginal thinking in the teaching of percutaneous transforaminal endoscopic discectomy. $J$ Reg Atan Oper Surg 2013;22:220-1. (in Chinese)

5. Liu FP, Zhao HW, Chen HD, Luo MY. Transforaminal endoscopic spine system for lumbar discectomy: the learning curve and our experiences. Orthop J China 2016;3:235-41. (in Chinese)

6. Wu XB, Fan GX, Gu X, Shen TG, Guan XF, Hu AN, Zhang HL, He SS. Learning curves of percutaneous endoscopic lumbar discectomy in transforaminal approach at the L4/5 and L5/S1 levels: a comparative study. J Zhejiang Univ Sci B 2016;17:553-60.

7. National Health and Family Planning Commission. Spinal Endoscopic Diagnosis and Treatment Management Standards (edition 2013). Available from: http://www.nhfpc.gov.cn/yzygj/s3585/201401/fd 7c01acb8b9465fa83abdeca4aed68a.shtml (the 5th attachment, in Chinese). [Last accessed on 6 Jul 2017] 